\title{
STABILITY OF BASES IN COMPLETE BARRELLED SPACES ${ }^{1}$
}

L. J. WEILL

1. Introduction. The main purpose of this paper is to prove for complete barrelled spaces a stability theorem, which is essentially a generalization of a stability theorem for Banach spaces obtained by B. E. Veĭc in [8].

Theorem. Let $E$ be a complete barrelled Hausdorff space with a Schauder basis $\left\{x_{k}\right\}$ bounded away from zero. If $\left\{y_{k}\right\}$ is an $\omega$-independent sequence in $E$ such that the series $\sum_{k=1}^{\infty} y_{k}-x_{k}$ converges unconditionally then $\left\{y_{k}\right\}$ is a Schauder basis for E equivalent to the basis $\left\{x_{k}\right\}$.

In the process of demonstrating this theorem we obtain some results which are of independent interest. Proposition 2, a generalization of Veřc's Banach space result [8], and Proposition 1 contain results which are not needed for the proof of the Theorem.

For the most part we shall follow the terminology of J. L. Kelley and I. Namioka in [5]. A barrel in a linear topological space is a set which is closed, convex, circled, and radial at zero. Banach spaces and Fréchet spaces (complete metrizable locally convex spaces) are special cases of barrelled spaces; i.e., locally convex spaces in which each barrel is a neighborhood of zero.

In a complete locally convex space $E$ a family $F$ of continuous linear functionals on $E$ is uniformly bounded on bounded subsets of $E$ provided that $F$ is pointwise bounded; in other words, weak* bounded subsets of $E^{*}$ are strongly bounded [5, p. 170]. A stronger property characterizes barrelled spaces; a locally convex space $E$ is barrelled if and only if a family $F$ of continuous linear functionals on $E$ is equicontinuous provided that it is pointwise bounded ( $[3$, the Barrel Theorem]; [5]). That is, $E$ is barrelled if and only if weak* bounded subsets of $E^{*}$ are equicontinuous.

2. In this section we will prove three seemingly unrelated propositions which will be needed for the proof of the theorem.

A basis in a linear topological space $E$ is a sequence $\left\{x_{k}\right\}$ in $E$ such that each element $x \in E$ has a unique representation $x=\sum_{k=1}^{\infty} a_{k} x_{k}$. The linear functionals $\left\{f_{k}\right\}$ defined by $f_{k}(x)=a_{k}$ are called the coeffi-

Received by the editors July 10, 1966 and, in revised form, October 4, 1966.

1 This work is taken from the author's dissertation (directed by C. W. McArthur) and was supported in part by NSF Grant GP-5952. 
cient functionals. When the coefficient functionals are continuous we call $\left\{x_{k}\right\}$ a Schauder basis. If a Banach space has a basis $\left\{x_{k}\right\}$ such that $\left\|x_{k}\right\|=1$ for each positive integer $k$, then the set of coefficient functionals is bounded (and equicontinuous). What can be said about the coefficient functionals in more general spaces with bases under similar conditions? The following proposition gives an answer.

Proposition 1. Let $E$ be a linear topological space with a Schauder basis $\left\{x_{k}\right\}$. Then each of the following statements holds.

(a) If $\left\{x_{k}\right\}$ is bounded away from zero then the family of coefficient functionals $\left\{f_{k}\right\}$ is weak* convergent to zero and therefore wea $k^{*}$ bounded.

(b) If $E$ is a complete locally convex space and $\left\{x_{k}\right\}$ is bounded away from zero then $\left\{f_{k}\right\}$ is strongly bounded.

(c) If $E$ is a barrelled space then $\left\{x_{k}\right\}$ is bounded away from zero if and only if $\left\{f_{k}\right\}$ is equicontinuous.

Proof. Since $\left\{x_{k}\right\}$ is a basis, the sequence $\left\{f_{k}(x) x_{k}\right\}$ converges to $\theta$ for each $x \in E$. Thus, since $\left\{x_{k}\right\}$ is bounded away from zero (i.e. $\theta$ is not a cluster point of $\left.\left\{x_{k}\right\}\right)$ the sequence $\left\{f_{k}(x)\right\}$ must converge to 0 , proving (a). (b) and the "only if" part of (c) follow immediately from (a) and the remarks made in the introduction. The proof of the "if" part of (c) is trivial and does not depend upon $E$ being barrelled.

Let $\left\{y_{k}\right\}$ be a sequence in a locally convex space $E$. The series $\sum_{k=1}^{\infty} y_{k}$ is said to converge unconditionally to an element $y$ of $E$ if for every permutation $p$ of the positive integers the series $\sum_{k=1}^{\infty} y_{p(k)}$ converges (in the usual sense) to $y$. T. H. Hildebrandt [4] proved that this is equivalent to unordered convergence which is defined in the following way. Let $S$ be the collection of finite subsets of the positive integers, ordered by $\supset$. Then $\sum_{k=1}^{\infty} y_{k}$ is unordered convergent to $y$, if the net $\left\{\sum_{k \in \sigma} y_{k}: \sigma \in S\right\}$ converges to $y$. It follows that if $U$ is a neighborhood of zero, there is an integer $N$ such that $\sum_{k \in \sigma} y_{k} \in U$, whenever $\sigma \in S$ and inf $\sigma \geqq N$. $\sum_{k=1}^{\infty} y_{k}$ is subseries convergent if for each subsequence $\left\{y_{k_{n}}\right\}$ of $\left\{y_{k}\right\}$, the series $\sum_{n=1}^{\infty} y_{k_{n}}$ is convergent. Subseries convergence is stronger than unconditional convergence but the two are equivalent in sequentially complete locally convex spaces. ${ }^{2}$

If $A$ is a subset of $E^{*}$ then the polar of $A$ (written $A_{0}$ ) in $E$ is the set $\{x \in E:|f(x)| \leqq 1$ for all $f \in A\}$. An important fact which is used in the next proposition is the following. The topology of a locally convex space $E$ is the topology of uniform convergence on equicontinuous

${ }^{2}$ For a more complete discussion see [2]. 
subsets of $E^{*}$; in other words $\left\{A_{0}: A\right.$ is an equicontinuous subset of $\left.E^{*}\right\}$ forms a local base for $E$. Define $a$ to be the set of sequences $\left\{\alpha_{n}\right\}$ such that $\alpha_{n}= \pm 1$ for each $n$, and define $e$ to be the set of all complex sequences $\left\{\epsilon_{n}\right\}$ such that $\left|\epsilon_{n}\right| \leqq 1$ for each $n$.

Proposition 2. Let $\left\{y_{k}\right\}$ be a sequence in a complete locally convex Hausdorff space E. The following statements are equivalent.

(1) The series $\sum_{k=1}^{\infty} y_{k}$ converges unconditionally.

(2) For any equicontinuous subset $A$ of $E^{*}$, the series $\sum_{k=1}^{\infty}\left|f\left(y_{k}\right)\right|$ converges uniformly with respect to $f \in A$.

(3) The series $\sum_{k=1}^{\infty} \epsilon_{k} y_{k}$ converges uniformly with respect to $\left\{\epsilon_{k}\right\} \in e$.

(4) The series $\sum_{k=1}^{\infty} \alpha_{k} y_{k}$ converges uniformly with respect to $\left\{\alpha_{k}\right\} \in a$.

Proof. The scheme of the proof is as follows: $(1) \rightarrow(2),(2) \rightarrow(3)$, $(3) \rightarrow(4),(4) \rightarrow(2)$, and (3) $\rightarrow(1)$. C. W. McArthur has shown directly that $(1) \leftrightarrow(2)$ in [6]. The implication $(3) \rightarrow(4)$ is trivial, and $(3) \rightarrow(1)$ is trivial since unconditional convergence is equivalent to subseries convergence in complete locally convex spaces.

$(1) \rightarrow(2)$. We shall use the following property of complex numbers. If $\left\{z_{k}\right\}$ is a sequence of complex numbers and $\left|\sum_{k \in \sigma} z_{k}\right|<M$ for all $\sigma \in S$ with inf $\sigma \geqq N$, then $\sum_{k=N}^{\infty}\left|z_{k}\right| \leqq 4 M$. Let $A$ be an equicontinuous subset of $E^{*}$ and $\epsilon$ a positive number. There is a neighborhood of zero $U$ of $E$ such that $|f(x)|<\epsilon / 5$ for all $x \in U$ and $f \in A$. Since $\sum_{k=1}^{\infty} x_{k}$ converges unconditionally there is an integer $N$ such that if $\sigma \in S$ and inf $\sigma \geqq N$ then $\sum_{k \in \sigma} x_{k} \in U$. Therefore $\left|f\left(\sum_{k \in \sigma} x_{k}\right)\right|$ $=\left|\sum_{k \in \sigma} f\left(x_{k}\right)\right|<\epsilon / 5$ for all $f \in A$. It follows from the above remark on complex sequences that $\sum_{\boldsymbol{k}=N}^{\infty}\left|f\left(x_{k}\right)\right|<\epsilon$ for all $f \in A$. Thus $\sum_{\boldsymbol{k}=1}^{\infty}\left|f\left(x_{k}\right)\right|$ converges uniformly for $f \in A$.

$(2) \rightarrow(3)$. Let $A$ be an equicontinuous subset of $E^{*}$. Since $\sum_{\boldsymbol{k}=1}^{\infty}\left|f\left(y_{k}\right)\right|$ converges uniformly for $f \in A$, there is an $N$ such that for $n>m \geqq N, \sum_{k=m}^{n}\left|f\left(y_{k}\right)\right|<1$ for all $f \in A$. Now if $\left\{\epsilon_{k}\right\} \in e$ and $f \in A$ we have $\left|f \sum_{k=m}^{n} \epsilon_{k} y_{k}\right| \leqq \sum_{k=m}^{n}\left|\epsilon_{k}\right| \cdot\left|f\left(y_{k}\right)\right| \leqq \sum_{k=m}^{n}\left|f\left(y_{k}\right)\right|<1$. Thus for $n>m \geqq N$, and any sequence $\left\{\epsilon_{k}\right\}$ in $e, \sum_{k=m}^{n} \epsilon_{k} y_{k} \in A_{0}$. Since $E$ is complete (3) is proved.

$(4) \rightarrow(2)$. Using again the fact that the topology of $E$ is the topology of uniform convergence on equicontinuous sets, and observing that for each complex number $t,|\operatorname{Re} t| \leqq|t|$ and $|\operatorname{Im} t| \leqq|t|$ we see that (4) implies: for each equicon tinuous subset $B$ of $E^{*}, \sum \alpha_{k} \operatorname{Re} f\left(y_{k}\right)$ and $\sum \alpha_{k} \operatorname{Im} f\left(y_{k}\right)$ converge uniformly for $\left\{\alpha_{k}\right\} \in a$ and $f \in B$. Let $\epsilon$ be positive and $A$ equicontinuous and pick $N$ large enough so that $n>m \geqq N$ implies $\left|\sum_{k=m}^{n} \alpha_{k} \operatorname{Re} f\left(y_{k}\right)\right| \leqq \epsilon / 2$ and $\left|\sum_{k=m}^{n} \alpha_{k} \operatorname{Im} f\left(y_{k}\right)\right|$ $\leqq \epsilon / 2$ for any $\left\{\alpha_{k}\right\} \in a$ and $f \in A$. Now let $f \in A$ and define the sequences $\left\{b_{k}\right\}$ and $\left\{c_{k}\right\}$ of $a$ by $\left|\operatorname{Re} f\left(y_{k}\right)\right|=b_{k} \operatorname{Re} f\left(y_{k}\right)$ and $\left|\operatorname{Im} f\left(y_{k}\right)\right|$ 
$=c_{k} \operatorname{Im} f\left(y_{k}\right)$. Then for $n>m \geqq N$ we have

$$
\begin{aligned}
\sum_{k=m}^{n}\left|f\left(y_{k}\right)\right| & \leqq \sum_{k=m}^{n}\left|\operatorname{Re} f\left(y_{k}\right)\right|+\left|\operatorname{Im} f\left(y_{k}\right)\right| \\
& =\sum_{k=m}^{n} b_{k} \operatorname{Re} f\left(y_{k}\right)+\sum_{k=m}^{n} c_{k} \operatorname{Im} f\left(y_{k}\right)<\epsilon .
\end{aligned}
$$

This completes the proof of the proposition.

A subset $C$ of a linear topological space $E$ is said to be relatively compact if it has compact closure. $C$ is relatively compact if and only if each net in $C$ has a cluster point in $E$. A linear operator $T: E \rightarrow F$ is called totally bounded if it maps bounded sets to totally bounded sets; compact if it maps bounded sets to relatively compact sets; and completely continuous if it maps a neighborhood of zero to a relatively compact set. If $F$ is a complete locally convex space the first two operators are equivalent. If $E$ is a normed space, the second two are equivalent. Thus the three types of operators are equivalent whenever both $E$ and $F$ are Banach spaces. In the general theory the strongest type, complete continuity, seems to be the most useful. We have the following proposition which implies a well-known property of compact operators in Banach spaces.

Proposition 3. Let $T, T_{1}, T_{2}, \cdots$, be linear operators on a linear topological space $E$ to a complete locally convex space $F$ such that $\left\{T_{n}\right\}$ converges to $T$ uniformly on a neighborhood of zero $V$ of $E$. If for each integer $n, T_{n}(V)$ is totally bounded then $T$ is completely continuous.

Proof. In a complete locally convex space a set is totally bounded if and only if it is relatively compact; hence we need only show that $T(V)$ is totally bounded. The proof is straightforward and is omitted.

3. Two bases $\left\{x_{k}\right\}$ and $\left\{y_{k}\right\}$ are said to be equivalent if for each sequence of scalars $\left\{a_{k}\right\}, \sum_{k=1}^{\infty} a_{k} x_{k}$ converges if and only if $\sum_{k=1}^{\infty} a_{k} y_{k}$ converges. A sequence $\left\{y_{k}\right\}$ is $\omega$-independent if the equation $\sum_{k=1}^{\infty} a_{k} y_{k}=\theta$ implies $a_{k}=0$ for each integer $k$.

THEOREM. Let $E$ be a complete barrelled Hausdorff space with a Schauder basis $\left\{x_{k}\right\}$ which is bounded away from zero. If $\left\{y_{k}\right\}$ is an $\omega$-independent sequence in $E$ such that the series $\sum_{\boldsymbol{k}=1}^{\infty}\left(y_{k}-x_{k}\right)$ converges unconditionally then $\left\{y_{k}\right\}$ is a Schauder basis for E equivalent to the basis $\left\{x_{k}\right\}$.

The proof depends upon several lemmas, the first three of which assume the hypothesis of the theorem. Let $\left\{f_{k}\right\}$ be the sequence of coefficient functionals associated with the basis $\left\{x_{k}\right\}$ of the Theorem. 
Lemma 1. There is a neighborhood of zero $V$ in $E$ such that the series $\sum_{k=1}^{\infty} f_{k}(x)\left(y_{k}-x_{k}\right)$ converges uniformly for $x \in V$ (and thus pointwise everywhere).

Proof. By Proposition 1, $\left\{f_{k}\right\}$ is equicontinuous so there exists a neighborhood of zero $V$ in $E$ such that $\left|f_{k}(x)\right|<1$ for all $x \in V$. If $A$ is an equicontinuous subset of $E^{*}$, then by Proposition $2((\mathrm{a}) \rightarrow(\mathrm{b}))$ there is a positive integer $N(A)$ such that $\sum_{k=m}^{n}\left|g\left(y_{k}-x_{k}\right)\right|<1$ for $n>m \geqq N$ and all $g \in A$. For $x \in V$ we have

$$
\begin{aligned}
\left|g\left(\sum_{k=m}^{n} f_{k}(x)\left(y_{k}-x_{k}\right)\right)\right| & \leqq \sum_{k=m}^{n}\left|f_{k}(x)\right| \cdot\left|g\left(y_{k}-x_{k}\right)\right| \\
& \leqq \sum_{k=m}^{n}\left|g\left(y_{k}-x_{k}\right)\right|<1 .
\end{aligned}
$$

Thus we have proved that for an equicontinuous subset $A$ of $E^{*}$ there is an integer $N(A)$ such that if $n>m \geqq N$ then

$$
\sum_{k=m}^{n} f_{k}(x)\left(y_{k}-x_{k}\right) \in A_{0} \quad \text { for all } x \in V .
$$

Thus since $E$ is complete we have proved that $\sum_{k=1}^{\infty} f_{k}(x)\left(y_{k}-x_{k}\right)$ converges uniformly for $x \in V$.

Lemma 2. For each positive integer $n$, define the linear operator $T_{n}$ by $T_{n}(x)=\sum_{k=1}^{n} f_{k}(x)\left(y_{k}-x_{k}\right)$. If $V$ is the neighborhood of Lemma 1 then $T_{n}(V)$ is relatively compact for each $n$.

Proof. Since the range of each $T_{n}$ is finite dimensional it suffices to show that $T_{n}(V)$ is bounded. Let $U$ be a neighborhood of zero in $E$ and $W$ a circled neighborhood of zero such that $W+W+\cdots$ $+W \subset U$, where there are $n W^{\prime}$ 's. For each integer $k$ in $[1 ; n]$ there is a scalar $a_{k}$ such that $y_{k}-x_{k} \in a_{k} W$. If $x \in V$ then $f_{k}(x)\left(y_{k}-x_{k}\right) \in a_{k} W$ because $a_{k} W$ is circled. Therefore if $x \in V$ we have

$$
T_{n}(x)=\sum_{k=1}^{n} f_{k}(x)\left(y_{k}-x_{k}\right) \in \sum_{k=1}^{n} a_{k} W \subset b U .
$$

where $b=\max \left\{\left|a_{1}\right|, \cdots,\left|a_{n}\right|\right\}$.

From Proposition 3, Lemma 1, and Lemma 2 we have the following.

Lemma 3. The linear operator $T$ defined by $T(x)=\sum_{k=1}^{\infty} f_{k}(x)\left(y_{k}-x_{k}\right)$ is completely continuous.

We state one more lemma which is a result of the abstract Riesz theory of completely continuous operators (see [7, sections 76-80], or more concisely [5, p. 207]). 
Lemma 4. Let $T$ be a completely continuous operator on a linear topological Hausdorff space E, and I the identity map on E. If $\lambda$ is not an eigenvalue of $T$ then the operator $T-\lambda I$ is a topological isomorphism of E onto E.

Proof of the Theorem. We may define a linear operator $S$ on $E$ by

$$
S(x) \equiv \sum_{k=1}^{\infty} f_{k}(x) y_{k}=\sum_{k=1}^{\infty} f_{k}(x)\left(y_{k}-x_{k}\right)+\sum_{k=1}^{\infty} f_{k}(x) x_{k}=T(x)+x .
$$

If $I$ is the identity operator on $E$ then we have that $S=T+I$. The sequence $\left\{y_{k}\right\}$ is $\omega$-independent so $S(x) \equiv \sum_{k=1}^{\infty} f_{k}(x) y_{k}=\theta$ implies that $x=\theta$. We may write $S=T-(-1) I$ and so it is clear that $\lambda=-1$ is not an eigenvalue for $T$. From Lemma 4 it follows that $S(x)$ $\equiv \sum_{\boldsymbol{k}=1}^{\infty} f_{k}(x) y_{k}$ is a topological isomorphism of $E$ onto $E$ and thus $\left\{y_{k}\right\}$ is a basis equivalent to $\left\{x_{k}\right\}$. If $\left\{g_{k}\right\}$ is the family of coefficient functionals for $\left\{y_{k}\right\}$ then it is clear that $g_{k}=f_{k} \circ S^{-1}$ and hence $\left\{y_{k}\right\}$ is a Schauder basis.

We make one last remark. An evaluable space (quasi-barrelled space) is a locally convex space $E$ such that the canonical mapping from $E$ to $E^{* *}$ is continuous. Every barrelled space is evaluable. In the statement of the theorem the word "barrelled" can be replaced by "evaluable" since it is true that a space is complete and barrelled if and only if it is complete and evaluable.

\section{BIBLIOGRAPHY}

1. N. Bourbaki, Espaces vectoriels topologiques, Actualités Sci. Ind., Nos. 1189, 1229, Hermann, Paris, 1953, 1955.

2. M. M. Day, Normed linear spaces, rev. ed., Academic Press, New York, 1962.

3. J. Dieudonné and L. Schwartz, La dualité dans les espaces $(F)$ et $(L F)$, Ann. Inst. Fourier (Grenoble) 1 (1949), 61-101.

4. T. H. Hildebrandt, On unconditional convergence in normed vector spaces, Bull. Amer. Math. Soc. 40 (1940), 959-962.

5. J. L. Kelley, I. Namioka, et al., Linear topological spqces, Van Nostrand, Princeton, N. J., 1963.

6. C. W. McArthur, A convergence criterion with applications to series in locally convex spaces, Duke Math. J. 34 (1967), 193-199.

7. F. Riesz and B. Sz-Nagy, Functional analysis, Ungar, New York, 1955.

8. B. E. Verc, Some stability properties of bases, Soviet Math. Dokl. 5 (1964), 1141-1144.

Florida State University 